\title{
Microscopic Selection of Solutions to Scalar Conservation Laws with Discontinuous Flux in the Context of Vehicular Traffic
}

\author{
Boris Andreianov (D) and Massimiliano D. Rosini (D)
}

\begin{abstract}
In the context of road traffic modeling we consider a scalar hyperbolic conservation law with the flux (fundamental diagram) which is discontinuous at $x=0$, featuring variable velocity limitation. The flow maximization criterion for selection of a unique admissible weak solution is generally admitted in the literature, however justification for its use can be traced back to the irrelevant vanishing viscosity approximation. We seek to assess the use of this criterion on the basis of modeling proper to the traffic context. We start from a first order microscopic follow-the-leader (FTL) model deduced from basic interaction rules between cars. We run numerical simulations of FTL model with large number of agents on truncated Riemann data, and observe convergence to the flow-maximizing Riemann solver. As an obstacle towards rigorous convergence analysis, we point out the lack of order-preservation of the FTL semigroup.
\end{abstract}

Keywords Conservation laws $\cdot$ Traffic flow $\cdot$ Discontinuous flux $\cdot$ Riemann solvers $\cdot$ First order follow-the-leader model $\cdot$ Order-preservation $\cdot$ Point constraint on the flux $\cdot$ Follow-the-leader semigroup

\footnotetext{
MDR is member of GNAMPA. MDR acknowledges the support of the National Science Centre, Poland, Project "Mathematics of multi-scale approaches in life and social sciences" No. 2017/25/B/ST1/00051, by the INdAM-GNAMPA Project 2019 "Equazioni alle derivate parziali di tipo iperbolico o non locale ed applicazioni" and by University of Ferrara, FIR Project 2019 "Leggi di conservazione di tipo iperbolico: teoria ed applicazioni".

\author{
B. Andreianov \\ Institut Denis Poisson (CNRS UMR7013), Université de Tours, Université d'Orléans, 37200 \\ Tours, Parc Grandmont, France \\ e-mail: boris.andreianov@univ-tours.fr
}

Peoples Friendship University of Russia (RUDN University), 6 Miklukho-Maklaya St, Moscow 117198, Russian Federation

\author{
M. D. Rosini ( $\varangle)$ \\ Department of Mathematics and Computer Science, University of Ferrara, I-44121 Ferrara, Italy \\ e-mail:rsnmsm@unife.it
}

Instytut Matematyki, Uniwersytet Marii Curie-Skłodowskiej, pl. Marii Curie-Skłodowskiej 1, 20-031 Lublin, Poland

J. Banasiak et al. (eds.), Semigroups of Operators - Theory and Applications,

Springer Proceedings in Mathematics \& Statistics 325,

https://doi.org/10.1007/978-3-030-46079-2_7 


\section{Introduction}

\subsection{Discontinuous-Flux Scalar Conservation Law}

The main object of study in this paper is the first order macroscopic model for vehicular traffic

$$
\partial_{t} \rho+\partial_{x} f(\rho, x)=0, \quad f(\rho, x) \doteq \begin{cases}f_{-}(\rho) & \text { if } x<0 \\ f_{+}(\rho) & \text { if } x \geqslant 0\end{cases}
$$

where $\rho=\rho(t, x) \in[0,1]$ is the (normalized) density at time $t \geqslant 0$ and position $x \in$ $\mathbb{R}$, while $f_{-} \geqslant 0$ and $f_{+} \geqslant 0$ are the fundamental diagrams (fluxes) corresponding to the two sections $x<0$ and $x>0$ of the road separated by $x=0$, called "interface" in the sequel. We assume that $f_{-}$and $f_{+}$are convex and satisfy the matching conditions

$$
f_{-}(0)=f_{+}(0), \quad f_{-}(1)=f_{+}(1) .
$$

Our concern is with admissibility of solution discontinuities at the interface location $x=0$, which is a question asked also for other models of vehicular traffic with point singularities, such as [14, 34, 54]. Our paper shares this primary motivation with the recent work [51]. The author of [51] studies admissible jumps at the flux discontinuity location by analyzing (both analytically and numerically) stationary wave profiles. We pursue (numerically only, at the present stage) the complementary line of investigation, by looking at solutions of the Riemann problems and interpreting our results in terms of global properties of the flow (we put forward the flux maximization principle).

Equation (1) is a scalar hyperbolic conservation law in one space dimension. It arises in road traffic but also in many other application problems, such as two-phase flows through porous media [5, 36], continuous sedimentation in a clarifier-thickener unit $[16,32]$ or ion etching in the semiconductor industry [49].

The derivatives in (1) are interpreted in the weak sense. Indeed, even for smooth data classical solutions may not exist globally in time since discontinuities can arise in finite time. Yet weak solutions are in general not unique. This motivates the introduction of additional admissibility criteria able to single out a unique solution. In principle, these conditions depend on the physical phenomena under consideration. However it turns out that in the setting of continuous flux (the case $f_{-} \equiv f_{+}$in (1)) the same notion of entropy admissible solution ([41], see also [42, 46, 55]) is relevant for all traditional applications. In different fluid mechanics applications the widely accepted justification for the choice of this solution notion is based upon the vanishing viscosity method that can be traced back as far as [37, 47]. The use of the same solution notion in the road traffic (the Lighthill-Whitham-Richards model [44, 48], LWR model in the sequel) is rather based upon the coincidence of admissible 
discontinuities: those observed in the traffic context turn out to be the admissible jumps in the entropy sense $[41,46]$.

The situation is drastically different in the discontinuous-flux setting of (1). Coexistence of infinitely many admissibility criteria-equally consistent from the pure mathematical standpoint-has been discovered in [1] and further developed e.g. in $[5,6,10,17,35]$. To be precise, there exist infinitely many $\mathbf{L}^{\mathbf{1}}$-contractive semigroups of solutions to the formal problem (1), moreover, different semigroups correspond to different modeling contexts or different model parameters. It is highlighted in the recent survey paper [4] that choosing admissibility condition for (1) at the interface $x=0$ means prescribing an interface coupling condition; this is very much similar to prescribing different boundary conditions (Dirichlet, Robin, Neumann, obstacle, etc.) for classical elliptic or parabolic PDEs. As a matter of fact, interface coupling conditions are part of the model and have to be prescribed independently from the differential relations applied in open space-time domains. E.g. in the context of flows in "two-rocks" porous media, the physically relevant coupling depends on additional nonlinearities $\phi_{-}, \phi_{+}$accounting for capillary pressures that are not even present in the formal PDE formulation (1), see [5]. Thus, considering (1) without precise indication of the desired interface coupling means working with an incomplete model, similar to speaking of "Laplacian operator on a bounded domain" without specific boundary conditions!

In the context of hyperbolic models of road traffic, many nontrivial models are postulated by prescribing Riemann solvers. The choice of admissibility conditions at $x=0$, i.e. the prescription of precise interface coupling conditions, is equivalent to the choice of a Riemann solver at the interface location [10, 35]. Making this choice is not always straightforward. In the traffic context, the lack of an established method to single out physically reasonable discontinuities leads to the introduction of different notions of solutions for the same problem (sometimes in the same paper!), see for instance [14, 34, 54]. In [35], it is even stated that "there is no a priori preferable physical solution". In this respect, let us stress that the vanishing viscosity (or, more generally, vanishing capillarity, cf. [5]) admissibility criterion is successful in fluid dynamics applications, but it is not recognized as instrumental in the context of traffic modeling.

\subsection{The Flow Maximization Principle, Models with a Point Constraint on the Flux and Their Justification}

There is a number of works on trafic modeling where the jump admissibility criterion is taken according to the principle of flow maximization: we refer, for instance, to $[18,20,26,40,45]$. It also appeared in modeling of flows in porous media [1, 5, $19,38]$, the resulting solution being referred to as the "optimal entropy solution" and justified via the vanishing capillarity approach in specific flux configurations. 
The flow maximization principle is appealing but not always justified; as a matter of fact, quite often it is implicitly assumed. This naturally occurs when one takes a successful-e.g., having a well-developed well-posedness theory-solution notion from the related literature, even if it may actually address a quite different application context. A further motivation stems from the fact that one and the same notion of solution was recognized as the correct one for very different applications, at least in the classical continuous-flux case of (1). However, we have seen that somewhat unexpectedly, the discontinuous-flux setting reveals much more diversity. From the accurate modeling perspective, in traffic applications the flow maximization should result as a collective behavior emerging from individual-driver behavior. However, flow maximization does not always occur in practice, as it is evident in the capacity drop phenomenon at crossroads [52] and in the Braess paradox phenomenon [15]. In particular, it was demonstrated in [7] that the capacity drop and the Braess paradox can be reproduced starting from the concept of flux limitation (as opposed to the flow maximization) introduced in $[21,22]$.

Let us briefly focus on the flux limitation model of [21]. It formally takes the form (1) with continuous flux (i.e., $f_{-}=f_{+} \doteq f$ ) and with explicit (though formal) interface condition $f\left(\rho\left(t, 0^{ \pm}\right)\right) \leqslant F(t)$ for some map $F$ given beforehand. This model is introduced essentially in terms of the intuitively appealing Riemann solver at the interface $x=0$; but the authors of [21] also provide a global entropy formulation and deduce it from the asymptotic analysis involving a discontinuous-flux conservation law similar to (1), but with two flux discontinuities situated at $x= \pm \varepsilon$, with $\varepsilon \rightarrow 0^{+}$. Doing so, they rely upon the global entropy formulation for (1) appearing in the influential works for the case where the graphs $f_{-}, f_{+}$can be ordered, such as $[39,50,53]$. However, the admissibility formulation in use in $[39,50,53]$ can be traced back to — or connected to, see e.g. [11,33]—-the vanishing viscosity approximation, not suitable for road traffic applications. Another source of justification is the "minimal jump" condition of [36], which relies on a mathematical rather than on a modeling selection principle. As a matter of fact, a byproduct of our analysis is a more realistic justification of the model introduced in [21].

We point out that at the present stage our justification is not rigorous because our conclusions are based only upon accurate modeling and numerical experiments lacking, at the present stage, the full convergence proof.

\subsection{Microscopic-to-Macroscopic Limit in Traffic Modeling. the Purpose and the Contents of the Paper}

Our main goal is to propose a method to select an appropriate solution. We rely on the paradigm of passage to the limit from a well-assessed microscopic model to a macroscopic model, similar to taking the hydrodynamical limit of Boltzmann equations. For the LWR traffic model, such passage to the limit was fully realized for the first time in [29]. The main advantage of this approach is that it requires to 
set assumptions on the interacting behavior of the cars only at the microscopic level. Since traffic dynamics are essentially microscopic, it is much easier to physically motivate the microscopic rather than some macroscopic assumptions. Thus, we first encode the interacting rules in a (first order) microscopic follow-the-leader (FTL) approximation of (1). We then obtain a macroscopic Riemann solver as many particle limit by applying, at the level of numerical simulations and for carefully identified sets of data, the approximation procedure adapted from [29]. It turns out that from this procedure, we indeed observe the emergence of the Riemann solver which maximizes the flow at $x=0$. Let us stress that we do not require this property at the microscopic level, as we only prescribe elementary car interaction rules. Therefore our results further assess the relevance of the flow maximization admissibility criterion, at least in the present setting of discontinuous-flux LWR model (1), in the related LWR model with point constraint on the flow and its variants [8, 9, 21]. A closely related investigation of the FTL approximation of the discontinuous-flux LWR model, with a focus on emerging standing-wave profiles (which is a way of understanding admissibility of discontinuities), is conducted in [51]; our results are complementary of those of [51].

Further, we identify specific difficulties on the way of rigorously proving the observed numerical convergence results. First, the techniques of [29] and subsequent works $[27,28,30,31]$ on the same subject do not extend readily to discontinuousflux LWR model (1). Second, note that while dealing with discontinuous-flux scalar conservation laws, common techniques of passage to the limit arose that are equally applicable to vanishing viscosity (or vanishing capillarity), Finite Volume and WaveFront Tracking approximations. These techniques are essentially based upon identification of a small number of explicit limits that take the form of stationary solutions, possibly discontinuous at $x=0$ (such discontinuities are therefore claimed admissible at the limit), and on the $\mathbf{L}^{\mathbf{1}}$-contraction property valid already at the level of approximations. We refer to [10] for a formalization of this technique. Note that the latter contraction principle is equivalent to the order-preservation of the solution semigroup, by virtue of the conservativity. We raise the question whether the FTL approximation of car densities fulfills the order-preservation property, and answer this question by a counterexample. This assesses the necessity of developing a nonstandard approach to the limit analysis of the FTL approximations. Moreover, while one expects on heuristic grounds that the admissibility at $x=0$ can be disconnected from admissibility (in the standard entropy sense) in the regions $\{x>0\},\{x<0\}$, the localization argument based on the finite speed of propagation in LWR models does not apply neither since the FTL model possess an infinite speed of propagation. We illustrate and discuss these two properties of the FTL semigroup, which make it qualitatively different from its expected limit which is the LWR model (1) with interface coupling prescribed by the flow maximization principle. 


\subsection{Outline of the Paper}

The paper is organized as follows. In the next section we introduce the model and the definition of admissible solutions corresponding to a Riemann solver in Definition 4. In Sect. 3 we recall the definition of the Riemann solver $\mathrm{RS}_{\mathrm{M}}$ which maximizes the flow at $x=0$ and then show how it can be deduced from a FTL model. In Sect. 4 we show that, differently from the macroscopic model, the microscopic model does not satisfy the order-preservation property and the finite speed of propagation. In the last section we explain how the Riemann solver introduced in [21] for a scalar conservation law with point constraint on the flow can be deduced from a FTL model.

\section{The Model and General Definition of Solutions}

Consider a road network parametrized by $x \in \mathbb{R}$ and composed of two single lane roads $\mathfrak{R}_{-} \doteq(-\infty, 0)$ and $\mathfrak{R}_{+} \doteq[0,+\infty)$ connected together at the junction $x=0$. Assume that each driver has the same behavior, all cars are identical and have maximal speed $V_{\max }>0$. Let $V_{-}, V_{+}>0$ be respectively the speed limits in $\Re_{-}$and $\Re_{+}$, with $V_{-}, V_{+} \leqslant V_{\max }$. The natural adaptation of the Lighthill, Whitham [44] and Richards [48] (LWR) model to the present case is expressed by the Cauchy problem for a one-dimensional scalar conservation law

$$
\begin{cases}\partial_{t} \rho+\partial_{x} f(\rho, x)=0, & x \in \mathbb{R}, t>0, \\ \rho(0, x)=\bar{\rho}(x), & x \in \mathbb{R},\end{cases}
$$

where $\rho=\rho(t, x) \in[0,1]$ is the (normalized) density of cars at time $t>0$ and position $x \in \mathbb{R}, \bar{\rho} \in \mathbf{L}_{\text {loc }}^{\mathbf{1}}(\mathbb{R} ;[0,1])$ is the initial density and the flux $f:[0,1] \times \mathbb{R} \rightarrow$ $[0,+\infty)$ is defined by

$$
f(r, x) \doteq \begin{cases}f_{-}(r) & \text { if } x \in \Re_{-} \\ f_{+}(r) & \text { if } x \in \Re_{+}\end{cases}
$$

where

$$
f_{-}(r) \doteq r v_{-}(r), \quad f_{+}(r) \doteq r v_{+}(r)
$$

Here $v_{-}, v_{+}:[0,1] \rightarrow\left[0, V_{\max }\right]$ are the velocity functions. We assume that $v_{-}$and $v_{+}$are continuous non-increasing functions with

$$
v_{-}(0)=V_{-}, \quad v_{+}(0)=V_{+}, \quad v_{-}(1)=0=v_{+}(1)
$$

and such that $f_{-}$and $f_{+}$are bell-shaped. Notice that $f(r, x)=r v(r, x)$, where $v:[0,1] \times \mathbb{R} \rightarrow\left[0, V_{\max }\right]$ is defined by 


$$
v(r, x) \doteq \begin{cases}v_{-}(r) & \text { if } x \in \mathfrak{R}_{-} \\ v_{+}(r) & \text { if } x \in \mathfrak{R}_{+}\end{cases}
$$

Typical choice for $v_{-}$and $v_{+}$is

$$
v_{-}(r) \doteq \min \left\{V_{-}, V_{\max }(1-r)\right\}, \quad v_{+}(r) \doteq \min \left\{V_{+}, V_{\max }(1-r)\right\}
$$

Since (2) is a conservation law, it is necessary to consider solutions in the sense of distributions, the so-called weak solutions. Define $\mathbb{R}_{+} \doteq[0,+\infty)$.

Definition 1 We say that $\rho \in \mathbf{C}^{\mathbf{0}}\left(\mathbb{R}_{+} ; \mathbf{B V}(\mathbb{R} ;[0,1])\right)$ is a weak solution to Cauchy problem (2) if the following conditions are satisfied:

(w.1) For any test function $\varphi \in \mathbf{C}_{\mathbf{c}}^{\infty}\left(\mathbb{R}_{+} \times \mathbb{R} ; \mathbb{R}\right)$ with compact support in $x<0$, we have

$$
\iint_{\mathbb{R}_{+} \times \mathfrak{R}_{-}}\left(\rho \partial_{t} \varphi+f_{-}(\rho) \partial_{x} \varphi\right) \mathrm{d} x \mathrm{~d} t+\int_{\mathfrak{R}_{-}} \bar{\rho}(x) \varphi(0, x) \mathrm{d} x=0 .
$$

(w.2) For any test function $\varphi \in \mathbf{C}_{\mathbf{c}}^{\infty}\left(\mathbb{R}_{+} \times \mathbb{R} ; \mathbb{R}\right)$ with compact support in $x>0$, we have

$$
\iint_{\mathbb{R}_{+} \times \mathfrak{R}_{+}}\left(\rho \partial_{t} \varphi+f_{+}(\rho) \partial_{x} \varphi\right) \mathrm{d} x \mathrm{~d} t+\int_{\mathfrak{R}_{+}} \bar{\rho}(x) \varphi(0, x) \mathrm{d} x=0 .
$$

(w.3) The Rankine-Hugoniot condition is satisfied along $x=0$, namely

$$
f_{-}\left(\rho\left(t, 0^{-}\right)\right)=f_{+}\left(\rho\left(t, 0^{+}\right)\right), \quad \text { for a.e. } t>0 .
$$

We stress that coupling condition (4) guarantees the conservation of the total number of cars.

Weak solutions are in general not unique. A well known criterion to select a unique weak solution was proposed by Kruzhkov [41]. However Kruzhkov solutions are not always physically reasonable [43]. This is evident, for instance, when dealing with traffic flows through crossroads [20, 25, 26, 35] or point-wise bottlenecks [21]. However, we stick to the notion of Kruzhkov admissibility away from the flux discontinuity location $x=0$. Namely, following [35] we postulate

Definition 2 We say that $\rho \in \mathbf{C}^{\mathbf{0}}\left(\mathbb{R}_{+} ; \mathbf{B V}(\mathbb{R} ;[0,1])\right)$ is an admissible solution to Cauchy problem (2) if it satisfies the following conditions:

(a.1) $\rho$ is a weak solution to Cauchy problem (2) in the sense of Definition 1.

(a.2) For any test function $\varphi \in \mathbf{C}_{\mathbf{c}}^{\infty}((0,+\infty) \times(-\infty, 0)$; $\mathbb{R})$ with $\varphi \geqslant 0$ and $\kappa \in$ $[0,1]$, we have 


$$
\iint_{\mathbb{R}_{+} \times \Re_{-}}\left(|\rho-\kappa| \partial_{t} \varphi+\operatorname{sign}(\rho-\kappa)\left(f_{-}(\rho)-f_{-}(\kappa)\right) \partial_{x} \varphi\right) \mathrm{d} x \mathrm{~d} t \geqslant 0
$$

(a.3) For any test function $\varphi \in \mathbf{C}_{\mathbf{c}}^{\infty}((0,+\infty) \times(0,+\infty)$; $\mathbb{R})$ with $\varphi \geqslant 0$ and $\kappa \in$ $[0,1]$, we have

$$
\iint_{\mathbb{R}_{+} \times \mathfrak{R}_{+}}\left(|\rho-\kappa| \partial_{t} \varphi+\operatorname{sign}(\rho-\kappa)\left(f_{+}(\rho)-f_{+}(\kappa)\right) \partial_{x} \varphi\right) \mathrm{d} x \mathrm{~d} t \geqslant 0 .
$$

Conditions listed in Definition 2 do not select a unique weak solution. However, to do so it is sufficient to uniquely characterize the physically reasonable discontinuities along $x=0$. This can be achieved by choosing a Riemann solver. Let $\mathbb{1}_{A}$ be the indicator function of the set $A$.

Definition 3 We say that RS: $[0,1]^{2} \rightarrow \mathbf{B V}(\mathbb{R} ;[0,1])$ is a Riemann solver if for any $\left(\rho_{L}, \rho_{R}\right) \in[0,1]^{2}$ the self-similar function

$$
\rho(t, x) \doteq \mathrm{RS}\left[\rho_{L}, \rho_{R}\right](x / t)
$$

together with its traces along $x=0$

$$
\rho^{-} \doteq \rho\left(t, 0^{-}\right)=\operatorname{RS}\left[\rho_{L}, \rho_{R}\right]\left(0^{-}\right), \quad \rho^{+} \doteq \rho\left(t, 0^{+}\right)=\operatorname{RS}\left[\rho_{L}, \rho_{R}\right]\left(0^{+}\right)
$$

satisfy the following conditions:

(r.1) $\rho$ is an admissible solution to Cauchy problem (2) with initial density

$$
\bar{\rho}(x) \doteq \rho_{L} \mathbb{1}_{\mathfrak{R}_{-}}(x)+\rho_{R} \mathbb{1}_{\mathfrak{R}_{+}}(x) .
$$

(r.2) The self-similar function

$$
r^{-}(t, x) \doteq \begin{cases}\rho(t, x) & \text { if } x<0, t \geqslant 0 \\ \rho^{-} & \text {if } x \geqslant 0, t \geqslant 0\end{cases}
$$

is the Kruzhkov solution to the Riemann problem

$$
\begin{cases}\partial_{t} r^{-}+\partial_{x} f_{-}\left(r^{-}\right)=0, & x \in \mathbb{R}, t>0 \\ r^{-}(0, x)=\rho_{L} \mathbb{1}_{\mathfrak{R}_{-}}(x)+\rho^{-} \mathbb{1}_{\mathfrak{R}_{+}}(x), & x \in \mathbb{R}\end{cases}
$$

namely for any test function $\varphi \in \mathbf{C}_{\mathbf{c}}^{\infty}\left(\mathbb{R}_{+} \times \mathbb{R} ; \mathbb{R}\right)$ with $\varphi \geqslant 0$ and $\kappa \in[0,1]$ 


$$
\begin{aligned}
& \iint_{\mathbb{R}_{+} \times \mathbb{R}}\left(\left|r^{-}-\kappa\right| \partial_{t} \varphi+\operatorname{sign}\left(r^{-}-\kappa\right)\left(f_{-}\left(r^{-}\right)-f_{-}(\kappa)\right) \partial_{x} \varphi\right) \mathrm{d} x \mathrm{~d} t \\
& \quad+\rho_{L} \int_{\mathfrak{R}_{-}} \varphi(0, x) \mathrm{d} x+\rho^{-} \int_{\mathfrak{R}_{+}} \varphi(0, x) \mathrm{d} x \geqslant 0 .
\end{aligned}
$$

(r.3) The self-similar function

$$
r^{+}(t, x) \doteq \begin{cases}\rho^{+} & \text {if } x<0, t \geqslant 0 \\ \rho(t, x) & \text { if } x \geqslant 0, t \geqslant 0\end{cases}
$$

is the Kruzhkov solution to the Riemann problem

$$
\begin{cases}\partial_{t} r^{+}+\partial_{x} f_{+}\left(r^{+}\right)=0, & x \in \mathbb{R}, t>0, \\ r^{+}(0, x)=\rho^{+} \mathbb{1}_{\mathfrak{R}_{-}}(x)+\rho_{R} \mathbb{1}_{\mathfrak{R}_{+}}(x), & x \in \mathbb{R}\end{cases}
$$

namely for any test function $\varphi \in \mathbf{C}_{\mathbf{c}}^{\infty}\left(\mathbb{R}_{+} \times \mathbb{R} ; \mathbb{R}\right)$ with $\varphi \geqslant 0$ and $\kappa \in[0,1]$

$$
\begin{aligned}
& \iint_{\mathbb{R}_{+} \times \mathbb{R}}\left(\left|r^{+}-\kappa\right| \partial_{t} \varphi+\operatorname{sign}\left(r^{+}-\kappa\right)\left(f_{+}\left(r^{+}\right)-f_{+}(\kappa)\right) \partial_{x} \varphi\right) \mathrm{d} x \mathrm{~d} t \\
& \quad+\rho^{+} \int_{\mathfrak{R}_{-}} \varphi(0, x) \mathrm{d} x+\rho_{R} \int_{\mathfrak{R}_{+}} \varphi(0, x) \mathrm{d} x \geqslant 0 .
\end{aligned}
$$

(r.4) We have

$$
\operatorname{RS}\left[\rho^{-}, \rho^{+}\right](\xi)= \begin{cases}\rho^{-} & \text {if } x<0 \\ \rho^{+} & \text {if } x \geqslant 0\end{cases}
$$

(r.5) The function $\left(\rho_{L}, \rho_{R}\right) \mapsto\left(f_{-}\left(\rho^{-}\right), f_{+}\left(\rho^{+}\right)\right)$is continuous.

Some comments on the conditions listed in Definition 3 are in order. Condition (r.1) ensures that a Riemann solver associates to any Riemann datum (6) a self-similar function $\rho$ through (5), which is an admissible solution to Riemann problem (2), (6). In particular it satisfies coupling condition (4)

$$
f_{-}\left(\rho^{-}\right)=f_{+}\left(\rho^{+}\right)
$$

Conditions (r.2), (r.3) can be rephrased as follows: $r^{-}$and $r^{+}$are the solutions to the initial-boundary value problems $[2,13,23]$

$$
\left\{\begin{array} { l l } 
{ \partial _ { t } r ^ { - } + \partial _ { x } f _ { - } ( r ^ { - } ) = 0 , } & { x < 0 , t > 0 , } \\
{ r ^ { - } ( 0 , x ) = \rho _ { L } , } & { x < 0 , } \\
{ r ^ { - } ( t , 0 ) = \rho ^ { - } , } & { t > 0 , }
\end{array} \quad \left\{\begin{array}{ll}
\partial_{t} r^{+}+\partial_{x} f_{+}\left(r^{+}\right)=0, & x>0, t>0 \\
r^{+}(0, x)=\rho_{R}, & x>0, \\
r^{+}(t, 0)=\rho^{+}, & t>0
\end{array}\right.\right.
$$


coupled through condition (8). Notice that $r^{-}$has only waves generated at $x=0$ and with negative speed, whereas $r^{+}$has only waves generated at $x=0$ and with positive speed. By (r.4) the ordered pair of the traces of the admissible solution selected by $R S$ is in a sense a fixed point of RS by (7). It is a minimal requirement to develop a numerical scheme with a time discretization based on RS, see [24, 25]. Condition (r.4) is therefore a stability condition. Condition (r.5) is a regularity property for RS.

We are now in the position to give the definition of admissible solutions to Cauchy problem (2) associated to a Riemann solver RS.

Definition 4 Fix a Riemann solver RS in the sense of Definition 3. We say that $\rho \in \mathbf{C}^{\mathbf{0}}\left(\mathbb{R}_{+} ; \mathbf{B V}(\mathbb{R} ;[0,1])\right)$ is an RS-admissible solution to Cauchy problem (2) if it is an admissible solution in the sense of Definition 2 and

$$
\operatorname{RS}\left[\rho\left(t, 0^{-}\right), \rho\left(t, 0^{+}\right)\right](\xi)=\left\{\begin{array}{l}
\rho\left(t, 0^{-}\right) \text {if } \xi<0, \\
\rho\left(t, 0^{+}\right) \text {if } \xi \geqslant 0,
\end{array} \text { for a.e. } t>0\right.
$$

In [35] it is proved that the above definition selects a unique admissible solution, given a fixed Riemann solver. We are therefore left to define a physically reasonable Riemann solver. This is achieved in the next section.

\section{Microscopic Deduction of the Riemann Solver $\mathbf{R S}_{M}$}

In this section we select a Riemann solver. The choice is motivated by basic well established physically reasonable rules on the microscopic interactions between the cars. We first deduce from the microscopic rules a FTL model. We then obtain an empirical Riemann solver as a many particle limit in analogy to what is done in [29]. It turns out that the obtained Riemann solver coincides with the Riemann solver $\mathrm{RS}_{\mathrm{M}}$ that maximizes the flow at $x=0$, even if we do not require any flow maximization property for the FTL model.

For completeness we recall in the next subsection the definition of $\mathrm{RS}_{\mathrm{M}}$. We then introduce the FTL model underlying the simulations which are used to show that the corresponding Riemann solver is indeed $\mathrm{RS}_{\mathrm{M}}$. In Figs. 1 and 2 we present the results of some selected simulations.

\subsection{Riemann Solver $\mathrm{RS}_{\mathrm{M}}$}

In this subsection we recall the Riemann solver $\mathrm{RS}_{\mathrm{M}}$ which maximizes the flow at $x=0$. This means that if RS is a Riemann solver, then by (8) 


$$
\begin{gathered}
f_{-}\left(\operatorname{RS}\left[\rho_{L}, \rho_{R}\right]\left(0^{-}\right)\right)=f_{+}\left(\operatorname{RS}\left[\rho_{L}, \rho_{R}\right]\left(0^{+}\right)\right) \\
\leqslant f_{-}\left(\operatorname{RS}_{\mathrm{M}}\left[\rho_{L}, \rho_{R}\right]\left(0^{-}\right)\right)=f_{+}\left(\operatorname{RS}_{\mathrm{M}}\left[\rho_{L}, \rho_{R}\right]\left(0^{+}\right)\right) .
\end{gathered}
$$

We introduce some notations. Let $L_{-}, L_{+}:[0,1]^{2} \rightarrow \mathbf{B V}(\mathbb{R} ;[0,1])$ be the Lax Riemann solvers associated to the fluxes $f_{-}$and $f_{+}$. Notice that $L_{-}\left[\rho_{L}, \rho_{R}\right] \equiv$ $\mathrm{L}_{+}\left[\rho_{L}, \rho_{R}\right]$ if and only if $\rho_{L}, \rho_{R} \geqslant \max \left\{R_{-}, R_{+}\right\}$, where $R_{-}, R_{+} \in(0,1)$ are implicitly defined by

$$
v\left(R_{-}\right)=V_{-}, \quad v\left(R_{+}\right)=V_{+} .
$$

Let $M_{-}$and $M_{+}$be the densities at which $f_{-}$and $f_{+}$reach their maxima. Let

$$
Q_{-}\left(\rho_{L}\right) \doteq\left\{\begin{array} { l l } 
{ f _ { - } ( \rho _ { L } ) } & { \text { if } \rho _ { L } < M _ { - } , } \\
{ f _ { - } ( M _ { - } ) } & { \text { if } \rho _ { L } \geqslant M _ { - } , }
\end{array} \quad Q _ { + } ( \rho _ { R } ) \doteq \left\{\begin{array}{l}
f_{+}\left(M_{+}\right) \text {if } \rho_{R}<M_{+}, \\
f_{+}\left(\rho_{R}\right) \text { if } \rho_{R} \geqslant M_{+},
\end{array}\right.\right.
$$

be the maximal flows at $x=0$ attained by $\mathrm{L}_{-}\left[\rho_{L}, \rho\right]$ and $\mathrm{L}_{+}\left[\rho, \rho_{R}\right]$ for $\rho$ varying in $[0,1]$. Let then $\hat{\rho}\left(\rho_{L}, \rho_{R}\right) \in\left[M_{-}, 1\right]$ and $\check{\rho}\left(\rho_{L}, \rho_{R}\right) \in\left[0, M_{+}\right]$be implicitly defined by

$$
f_{-}\left(\hat{\rho}\left(\rho_{L}, \rho_{R}\right)\right)=\min \left\{Q_{-}\left(\rho_{L}\right), Q_{+}\left(\rho_{R}\right)\right\}=f_{+}\left(\check{\rho}\left(\rho_{L}, \rho_{R}\right)\right) .
$$

Then $\mathrm{RS}_{\mathrm{M}}:[0,1]^{2} \rightarrow \mathbf{B V}(\mathbb{R} ;[0,1])$ is defined by

$$
\operatorname{RS}_{\mathrm{M}}\left[\rho_{L}, \rho_{R}\right](v) \doteq \begin{cases}\mathrm{L}_{-}\left[\rho_{L}, \hat{\rho}\left(\rho_{L}, \rho_{R}\right)\right](v) & \text { if } v<0 \\ \mathrm{~L}_{+}\left[\check{\rho}\left(\rho_{L}, \rho_{R}\right), \rho_{R}\right](v) & \text { if } v \geqslant 0\end{cases}
$$

\subsection{FTL Model}

In this subsection we introduce the FTL model corresponding to Riemann problem (2), (6). Fix $\left(\rho_{L}, \rho_{R}\right) \in[0,1]^{2}$ and consider the approximate Riemann problem

$$
\begin{cases}\partial_{t} \rho+\partial_{x} f(\rho, x)=0, & x \in \mathbb{R}, t>0, \\ \rho(0, x)=\rho_{L} \mathbb{1}_{[-\delta, 0)}(x)+\rho_{R} \mathbb{1}_{[0, \delta]}(x), & x \in \mathbb{R} .\end{cases}
$$

Here $\delta>0$ is introduced so that the traffic has finite total length of cars, which is $\delta\left(\rho_{L}+\rho_{R}\right)$. The case $\rho_{L}=0=\rho_{R}$ is trivial; assume therefore that $\rho_{L}+\rho_{R} \neq 0$. Let $\left[x_{\min }, x_{\max }\right]$ be the support of the initial datum, namely

$$
x_{\min } \doteq\left\{\begin{array} { l l } 
{ - \delta } & { \text { if } \rho _ { L } \neq 0 , } \\
{ 0 } & { \text { if } \rho _ { L } = 0 , }
\end{array} \quad x _ { \operatorname { m a x } } \doteq \left\{\begin{array}{ll}
\delta & \text { if } \rho_{R} \neq 0, \\
0 & \text { if } \rho_{R}=0 .
\end{array}\right.\right.
$$


In analogy with [29], for any fixed $n \in \mathbb{N}$, we let $\ell \doteq \delta\left(\rho_{L}+\rho_{R}\right) / n$ and consider the approximate discrete density

$$
\mathrm{r}(t, x) \doteq \sum_{i=1}^{n} \frac{\ell}{x_{i}(t)-x_{i+1}(t)} \mathbb{1}_{\left[x_{i+1}(t), x_{i}(t)\right)}(x),
$$

where $x_{i}$ are the solutions to the FTL model

$$
\left\{\begin{array}{lll}
\dot{x}_{1}=V_{+}, & t>0, & \\
\dot{x}_{i}=v\left(\frac{\ell}{x_{i-1}-x_{i}}, x_{i}\right), & t>0, & i \in\{2, \ldots, n+1\}, \\
x_{i}(0)=\bar{x}_{i}, & & i \in\{1, \ldots, n+1\} .
\end{array}\right.
$$

Above $\bar{x}_{1} \doteq x_{\max }, \bar{x}_{n+1} \doteq x_{\min }$ and for $i \in\{2, \ldots, n\}$ we define

$$
\begin{array}{ll}
\rho_{L} \neq 0 \Longrightarrow x_{i+1}^{0}=-\delta+(n-i) \frac{\ell}{\rho_{L}} \leqslant 0, & i \geqslant\left\lceil\frac{\rho_{R}}{\rho_{L}+\rho_{R}} n\right\rceil, \\
\rho_{R} \neq 0 \Longrightarrow x_{i+1}^{0}=\delta-i \frac{\ell}{\rho_{R}} \geqslant 0, & i \leqslant\left\lfloor\frac{\rho_{R}}{\rho_{L}+\rho_{R}} n\right\rfloor .
\end{array}
$$

Notice that by definition $\int_{\bar{x}_{i}}^{\bar{x}_{i-1}} \bar{\rho}(x) \mathrm{d} x=\ell$ and for any $t \geqslant 0$ we have

$$
\|\mathrm{r}(t)\|_{\mathbf{L}^{\mathbf{1}}(\mathbb{R})}=\delta\left(\rho_{L}+\rho_{R}\right), \quad x_{1}(t)=x_{\max }+V_{+} t
$$

We recall that (11) can be interpreted as a Lagrangian many particle approximation of (9). Moreover (11) corresponds to the following basic rules:

- Cars respect the speed limits.

- Cars do not overtake each other.

- Each car adjusts instantaneously its velocity according to the distance from its predecessor.

- Higher velocities correspond to higher distances from the predecessor.

In [29] it is proved that in the case $V_{-}=V_{+}=V_{\max }>0$ the discretized density $r$ given in (10) converges a.e. in $\mathbf{L}_{\text {loc }}^{\mathbf{1}}\left(\mathbb{R}_{+} \times \mathbb{R}\right)$ to the unique Kruzhkov [41] solution $\rho$ to (9). The rigorous proof for the convergence of the discretized density $r$ in the general case $V_{-} \neq V_{+}$is beyond the purposes of the present paper and is left to a future work. Here we are just interested in empirically deducing a Riemann solver from $\mathrm{ad}$ hoc computer assisted numerical simulations of (10), (11). It should be stressed that the simulations appear indeed as stable and convergent in all simulations we have run.

We are not interested in the solutions of (9) in the whole of $\mathbb{R}$, but only in the vicinity of $x=0$. For this reason we do not investigate the nature of the waves created at $x= \pm \delta$ and their interactions with the waves starting from $x=0$. 

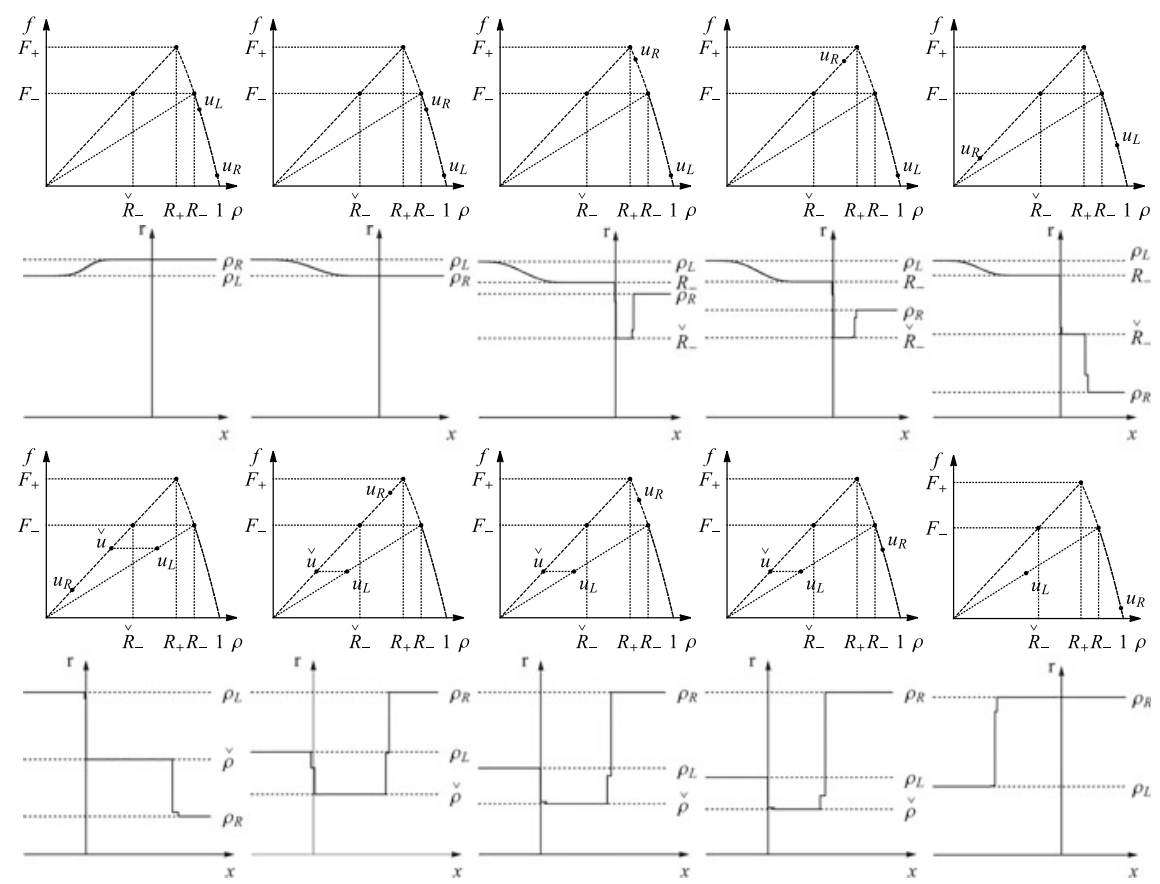

Fig. 1 Case $V_{-}<V_{+}<1 / 2$

Computer assisted numerical simulations allow to describe the limit solutions for any Riemann problem (2), (6). Our choice for the initial data $\left(\rho_{L}, \rho_{R}\right) \in[0,1]^{2}$ used for the simulations allows to encompass all the cases necessary to deduce a Riemann solver, which turns out to coincide with $\mathrm{RS}_{\mathrm{M}}$.

In Figs. 1 and 2 we present the outputs of the numerical simulations in the case $V_{-}, V_{+} \in(0,1 / 2)$; the remaining cases are analogous and are not furnished here not to overload the paper. In Figs. 1 and 2 we show $u_{L} \doteq\left(\rho_{L}, f_{-}\left(\rho_{L}\right)\right)$ and $u_{R} \doteq$ $\left(\rho_{R}, f_{+}\left(\rho_{R}\right)\right)$ in the fundamental diagram and below it we show the profile of the corresponding approximate discrete density $\mathrm{r}$ at a time $t>0$ of interest. For each simulation we take $n=1000, V_{\max }=1$ and $v_{-}, v_{+}$given by (3) with $V_{-}, V_{+} \in$ $\left\{\frac{2-\sqrt{2}}{4}, \frac{1}{4}\right\}$.

\section{Qualitative Comparison of LWR and FTL Models}

In this section we consider a homogeneous road and assume $V_{-}=V_{+}=V_{\max }$. In [29] it is proved that in this case the unique entropy solution to the LWR model is the limit of discrete densities corresponding to the FTL model. Despite this link between the two models, there are deep differences between them. We show below that the 

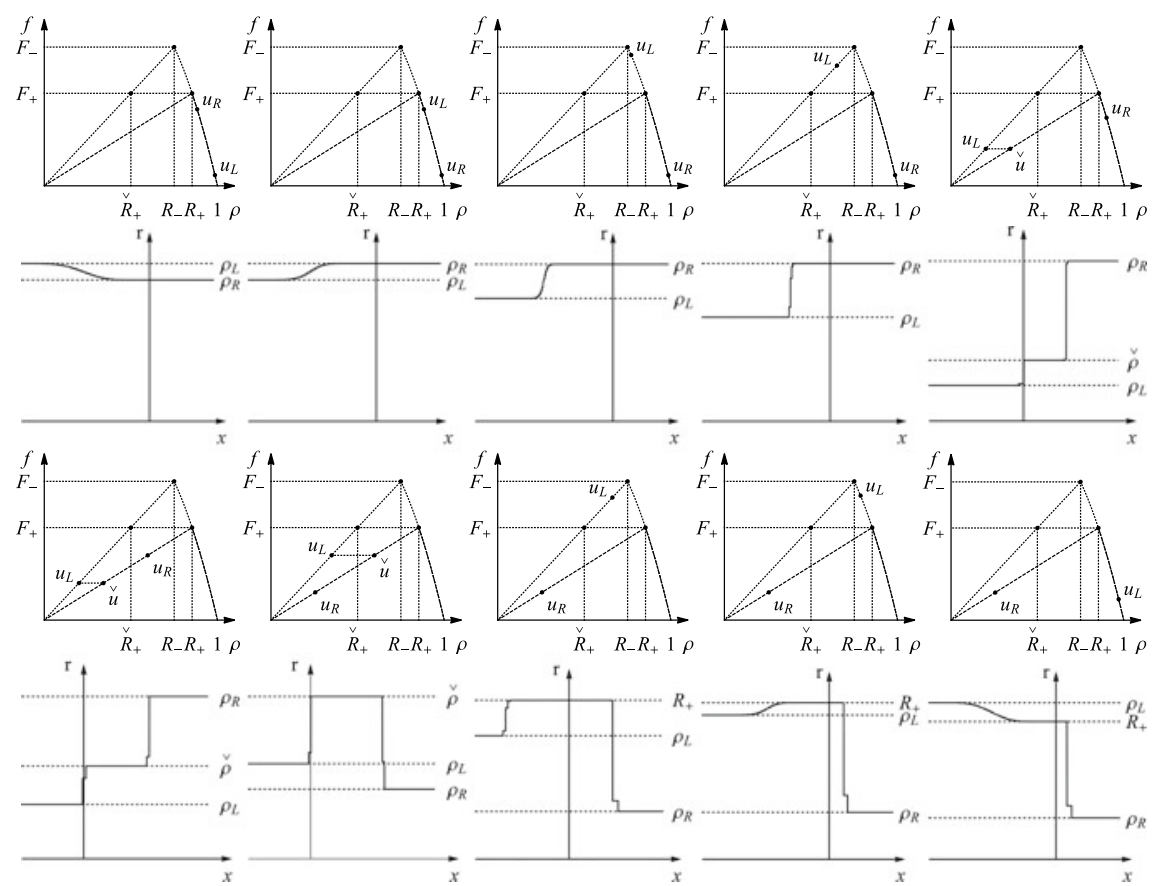

Fig. 2 Case $V_{+}<V_{-}<1 / 2$

order-preservation property and the finite speed of propagation, well known for the LWR model, are not shared by the FTL model. In a sense, these properties are gained only at the limit $n \rightarrow+\infty$.

We stress that the examples given below can be easily adapted to the case of inhomogeneous roads: it is enough to place all the cars on the right of $x=0$. In fact, properties of the discontinous-flux semigroup cannot be better than those of the continuous-flux one.

For simplicity consider a single lane road with neither entrances nor exits and parametrized by $x \in \mathbb{R}$. Let $\ell>0$ be the length of each car. The evolution of the traffic can be then described at the macroscopic level by the LWR model and at the microscopic level by the FTL model. The LWR model is expressed by the conservation law

$$
\partial_{t} \rho+\partial_{x} f(\rho)=0, \quad x \in \mathbb{R}, t>0
$$

where $\rho=\rho(t, x) \in[0,1]$ is the (normalized) density at time $t$ and position $x$, and $f(\rho) \doteq \rho v(\rho)$ is the flux corresponding to the velocity

$$
v(\rho) \doteq V_{\max }(1-\rho) .
$$


The FTL model is given by the system of ordinary differential equations

$$
\begin{cases}\dot{x}_{1}=V_{\max }, & t>0, \\ \dot{x}_{i}=v\left(\frac{\ell}{x_{i-1}-x_{i}}\right), & t>0, \quad i \in\{2, \ldots, n\},\end{cases}
$$

where $x_{i}=x_{i}(t) \in \mathbb{R}$ is the position at time $t$ of the front bumper of the $i$ th car labeled starting from the right and $n$ is the total number of cars. The corresponding discrete density is

$$
\mathrm{r}(t, x) \doteq \sum_{i=1}^{n-1} \frac{\ell}{x_{i}(t)-x_{i+1}(t)} \cdot \mathbb{1}_{\left[x_{i+1}(t), x_{i}(t)\right)}(x) .
$$

Notice that the total length of the traffic is $\ell n$ and is linked to the discrete density $r$ and the solution $\rho$ to LWR model (12) as follows:

$$
\int_{\mathbb{R}} \mathrm{r}(t, x) \mathrm{d} x+\ell=\ell n=\int_{\mathbb{R}} \rho(t, x) \mathrm{d} x, \quad t \geqslant 0 .
$$

\subsection{Finite Speed of Propagation}

It is well known that in the LWR model, information propagates with speed bounded by $V_{\max }$. On the contrary, according to the FTL model the propagation of information can be instantaneous. The next example shows that this drawback of the FTL model is responsible for an unrealistic behavior of the cars.

Example 1 Consider a traffic light placed at $x=0$, which at time $t=0$ turns from red to green. Assume that at time $t=0$ the cars in $x \leqslant 0$ are bumper to bumper while in $x>0$ no car is present. The corresponding FTL model (13) is

$$
\left\{\begin{array}{lll}
\dot{x}_{1}=V_{\max }, & t>0, & \\
\dot{x}_{i}=v\left(\frac{\ell}{x_{i-1}-x_{i}}\right), & t>0, & i \in\{2, \ldots, n\}, \\
x_{i}(0)=-(i-1) \ell, & & i \in\{1, \ldots, n\} .
\end{array}\right.
$$

We obviously have

$$
\begin{cases}x_{1}(t)=V_{\max } t, & t>0, \\ x_{i}(t)>-(i-1) \ell, & t>0, \quad i \in\{2, \ldots, n\} .\end{cases}
$$



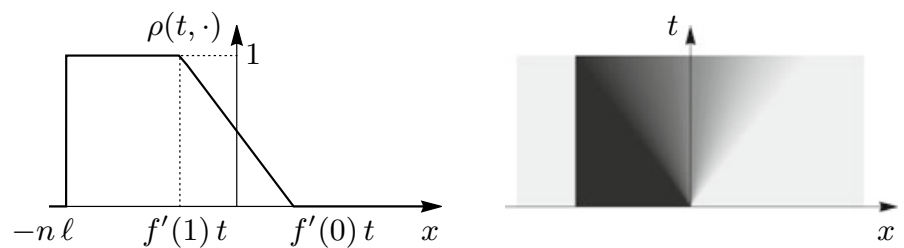

Fig. 3 Solution (18) for $t \in\left(0,-n \ell / f^{\prime}(1)\right)$. On the right, darker $(x, t)$-regions correspond to higher densities

The corresponding LWR model (12) is

$$
\begin{cases}\partial_{t} \rho+\partial_{x} f(\rho)=0, & x \in \mathbb{R}, t>0 \\ \rho(0, x)= \begin{cases}1 & \text { if } x \in[-n \ell, 0), \\ 0 & \text { otherwise },\end{cases} & x \in \mathbb{R}\end{cases}
$$

In the time interval $t \in\left(0,-n \ell / f^{\prime}(1)\right)$, its entropy solution $\rho$ is the juxtaposition of a stationary shock $\mathrm{S}(0,1)$ starting from $x=-n \ell$ and a rarefaction $\mathrm{R}(1,0)$ starting from $x=0$, namely

$$
\begin{aligned}
\rho(t, x) & = \begin{cases}1 & \text { if }-n \ell \leqslant x / t<f^{\prime}(1) \\
\left(f^{\prime}\right)^{-1}(x / t) & \text { if } f^{\prime}(1) \leqslant x / t<f^{\prime}(0) \\
0 & \text { otherwise }\end{cases} \\
& = \begin{cases}1 & \text { if }-n \ell \leqslant x / t<-V_{\max }, \\
\frac{1}{2}\left(1-\frac{\xi}{V_{\max }}\right) & \text { if }-V_{\max } \leqslant x / t<V_{\max }, \\
0 & \text { otherwise, }\end{cases}
\end{aligned}
$$

see Fig. 3.

From (18) we can deduce the corresponding trajectories of the cars. Indeed, if $x=X_{i}(t)$ is the trajectory of the $i$ th car, then

$$
\begin{cases}\dot{X}_{i}=v\left(\rho\left(t, X_{i}\right)\right), & t>0, \quad i \in\{1, \ldots, n\} \\ X_{i}(0)=-(i-1) \ell, & t>0, \quad i \in\{1, \ldots, n\}\end{cases}
$$

We obviously have

$$
\begin{cases}X_{1}(t)=V_{\max } t, & t>0 \\ X_{i}(t)=-i \ell, & t \in\left(0,-(i-1) \ell / f^{\prime}(1)\right), \quad i \in\{2, \ldots, n\}\end{cases}
$$

By comparing (16) and (20) it is clear that the trajectories corresponding to the microscopic (15) and macroscopic (17) descriptions do not coincide. Indeed, accord- 
Fig. 4 Comparison of microscopic (15) (dotted) and macroscopic (19) (dashed) trajectories

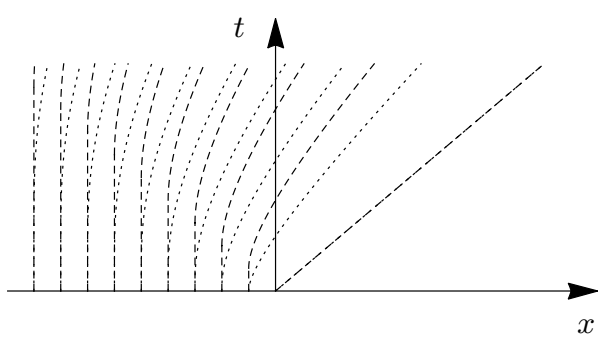

Fig. 5 The graphs of $r^{1}(0, \cdot)$ (dotted) and $\mathrm{r}^{2}(0, \cdot)$ (dashed) considered in Example 2



ing to the microscopic description each car start to move at time $t=0^{+}$with strictly positive speed. On the contrary, according to the macroscopic description, with exception for the rightmost car, all the other cars need some time before starting to move. In Fig. 4 we plot the trajectories corresponding to the two levels of descriptions.

Remark 1 We stress that real life experience shows that, at least in the case considered in Example 1, the macroscopic description is more appropriate than the microscopic one. Indeed, everyday experience shows that the cars don't start to move all together immediately after $t=0$.

\subsection{Order-Preservation Property}

In this subsection we consider the order-preservation property. It is well known that the LWR model satisfies it: if $\rho^{1}$ and $\rho^{2}$ are solutions to (12) corresponding to the initial data $\bar{\rho}^{1}$ and $\bar{\rho}^{2}$, and $\bar{\rho}^{1} \leqslant \bar{\rho}^{2}$, then also $\rho^{1} \leqslant \rho^{2}$. The next example shows that FTL model (13) is not order-preserving.

Example 2 Fix $0<\bar{x}_{3}^{1}<\bar{x}_{2}^{1}<\bar{x}_{1}^{1}$ and $0<\bar{x}_{4}^{2}<\ldots<\bar{x}_{1}^{2}$ as in Fig. 5 with

$$
\bar{x}_{3}^{2}-\bar{x}_{4}^{2}=\bar{x}_{2}^{1}-\bar{x}_{3}^{1}>\bar{x}_{2}^{2}-\bar{x}_{3}^{2}>\bar{x}_{1}^{2}-\bar{x}_{2}^{2}=\bar{x}_{1}^{1}-\bar{x}_{2}^{1}=\ell .
$$

By considering $\bar{x}_{3}^{1}, \bar{x}_{2}^{1}, \bar{x}_{1}^{1}$ and $\bar{x}_{4}^{2}, \ldots, \bar{x}_{1}^{2}$ as initial positions for FTL model (13), we obtain the discrete densities $r^{1}$ and $r^{2}$ via (14). By construction, at the initial time we have $\mathrm{r}^{1}(0, \cdot) \leqslant \mathrm{r}^{2}(0, \cdot)$. Clearly $\dot{x}_{3}^{1}(0)=\dot{x}_{4}^{2}(0)$ and $\dot{x}_{2}^{1}(0)=0<\dot{x}_{3}^{2}(0)$; thus, at least for $t>0$ sufficiently small, we have $x_{3}^{2}(t)-x_{4}^{2}(t)>x_{2}^{1}(t)-x_{3}^{1}(t)$ and therefore $\mathrm{r}^{1}(t, \cdot)>\mathrm{r}^{2}(t, \cdot)$ in the space interval $\left(x_{3}^{1}(t), x_{3}^{2}(t)\right)$.

Remark 2 It should be stressed that order-preservation implies $\mathbf{L}^{\mathbf{1}}$-contractivity by virtue of the classical Crandall-Tartar lemma. The semigroups of solutions of (1) 
considered in the present paper are $\mathbf{L}^{\mathbf{1}}$-contractive (cf. [3]), governed by accretive operators on $\mathbf{L}^{\mathbf{1}}$. As we can now see, the FTL semigroup (defined on densities) is conservative but not order-preserving: the FTL semigroup is not governed by an accretive operator on $\mathbf{L}^{\mathbf{1}}$. However, since the above example concerns the case with space-homogeneous flux, the convergence results of, e.g., [29] apply. Thus we observe that the approximation of (1), although convergent and motivated by modeling considerations, fails to share the key mathematical structure of the limit problem.

It is easily seen that the lack of order-preservation for the homogeneous FTL model underlying (12) is inherited by the FTL model underlying the discontinuousflux équation (1): indeed, its is enough to consider initial data supported in $\mathfrak{R}_{+}$.

Remark 3 In the context of (1), preservation by various approximation schemes of specific steady states is the cornerstone of the proofs of convergence of vanishing (or adapted) viscosity approximations, of Godunov Finite Volume approximations, or of the Wave-Front Tracking approximations to (1), see [4, 10]. In the context of fluxes of kind (3), it is not difficult to show that the FTL approximation does preserve the key steady state corresponding to the maximization of the interface flux. Yet, the absence of $\mathbf{L}^{\mathbf{1}}$-contraction at the FTL level precludes us from exploiting the convergence paradigm suitable for above mentioned order-preserving approximations. This leaves space to future work on rigorous justification of the convergence behavior reported in the present paper.

\section{Conservation Laws with Point Constraint on the Flow}

In this section we show how the above arguments justify the Riemann solver $\mathrm{RS}_{F}$ introduced in [21] for the scalar conservation law with point constraint on the flow

$$
\begin{cases}\partial_{t} \rho+\partial_{x} f(\rho)=0, & x \in \mathbb{R}, t>0 \\ \rho(0, x)=\bar{\rho}(x), & x \in \mathbb{R}, \\ f\left(\rho\left(t, 0^{ \pm}\right)\right) \leqslant F, & t>0\end{cases}
$$

Above $\rho \in[0,1]$ is again the (normalized) density of cars, $f:[0,1] \rightarrow\left[0, f_{\max }\right]$ is the flux and $F \in\left(0, f_{\max }\right)$ is the capacity of a pointwise bottleneck localized at $x=0$, e.g., a toll gate. We assume that the flux is bell shaped, attains its maximal value $f_{\max }>0$ at $R_{\max } \in(0,1)$ and takes the form $f(\rho)=\rho v(\rho)$, where the velocity function $v:[0,1] \rightarrow\left[0, V_{\max }\right]$ is a continuous non-increasing function such that $v(0)=V_{\max }$ and $v(1)=0$.

Since we are interested only in deducing a Riemann solver, we consider only Riemann initial conditions

$$
\bar{\rho}(x) \doteq \rho_{L} \mathbb{1}_{x<0}(x)+\rho_{R} \mathbb{1}_{x \geqslant 0}(x)
$$


As in [21] we introduce an approximate Riemann problem

$$
\begin{cases}\partial_{t} \rho+\partial_{x} f_{\varepsilon}(\rho, x)=0, & x \in \mathbb{R}, t>0, \\ \rho(0, x)=\bar{\rho}(x), & x \in \mathbb{R},\end{cases}
$$

where $f_{\varepsilon}:[0,1] \times \mathbb{R} \rightarrow\left[0, f_{\max }\right], \varepsilon>0$, is defined by

$$
f_{\varepsilon}(\rho, x) \doteq \begin{cases}f(\rho) & \text { if }|x| \geqslant \varepsilon \\ f_{F}(\rho) & \text { if }|x|<\varepsilon\end{cases}
$$

where $f_{F}(\rho) \doteq \rho \min \left\{V_{F}, v(\rho)\right\}$, with $V_{F} \in\left(0, V_{\max }\right)$ implicitly given by

$$
V_{F}=v\left(R_{F}\right), \quad R_{F} V_{F}=F, \quad R_{F}>R_{\max } .
$$

In words, we zoom at $x=0$, extend the location of the bottleneck to the interval $(-\varepsilon, \varepsilon)$, consider there a flux $f_{F}$ having $F$ as maximum value and corresponding to a velocity function of the form (3).

We then apply $\mathrm{RS}_{\mathrm{M}}$ at the discontinuity points $x= \pm \varepsilon$ of $f_{\varepsilon}$, construct the approximate solution $\rho_{\varepsilon}$, let $\varepsilon$ go to zero and obtain the limit function $\rho$. It is easy to seen then that $\rho \equiv \operatorname{RS}_{F}\left[\rho_{L}, \rho_{R}\right]$. For clarity, we consider in the next example one case.

Example 3 Fix $0<V<V_{\max }$ and consider the flux $f(r) \doteq r v(r)$ with

$$
v(r) \doteq \min \left\{V, V_{\max }(1-r)\right\}
$$

see Fig. 6. By definition $R_{\max }=1-\frac{V}{V_{\max }}$. Fix $\rho_{L}, \rho_{R} \in\left(0, R_{\max }\right)$ and assume that $f\left(\rho_{L}\right)>F$. The solution to (22), (23) can be constructed as follows. Introduce the following notation

$$
\begin{array}{lll}
u_{L} \doteq\left(\rho_{L}, f\left(\rho_{L}\right)\right), & u_{R} \doteq\left(\rho_{R}, f\left(\rho_{R}\right)\right), & U_{F} \doteq\left(R_{F}, F\right), \\
u_{L}^{\varepsilon} \doteq\left(\rho_{L}, f_{F}\left(\rho_{L}\right)\right), & u_{R}^{\varepsilon} \doteq\left(\rho_{R}, f F\left(\rho_{R}\right)\right), & \\
\check{u}_{L} \doteq\left(\check{\rho}_{L}, f\left(\check{\rho}_{L}\right)\right), & \check{u}_{R} \doteq\left(\check{\rho}_{R}, f\left(\check{\rho}_{R}\right)\right), & \check{U}_{F} \doteq\left(\check{R}_{F}, f\left(\check{R}_{F}\right)\right),
\end{array}
$$

where $\check{\rho}_{L}, \check{\rho}_{R}, \check{R}_{F} \in\left(0, R_{\max }\right)$ are implicitly defined by

$$
f\left(\check{\rho}_{L}\right)=f_{F}\left(\rho_{L}\right), \quad f\left(\check{\rho}_{R}\right)=f_{F}\left(\rho_{R}\right), \quad f\left(\check{R}_{F}\right)=f\left(R_{F}\right)=F .
$$

We first apply $\mathrm{RS}_{\mathrm{M}}$ at the discontinuity points $x= \pm \varepsilon$ of the flux $f_{\varepsilon}$. At $x=-\varepsilon$ we obtain the shock $\mathrm{S}\left(u_{L}, U_{F}\right)$ with negative speed (of propagation) and the contact discontinuity $\operatorname{CD}\left(U_{F}, u_{L}^{\varepsilon}\right)$ with speed $V_{F}$. At $x=\varepsilon$ we obtain the (stationary) nonclassical shock NS $\left(u_{R}^{\varepsilon}, \breve{u}_{R}\right)$ and $\operatorname{CD}\left(\breve{u}_{R}, u_{r}\right)$ with speed $V$. At $x=0$ we apply the Lax Riemann solver associated to the flux $f_{F}$ and obtain $\operatorname{CD}\left(u_{L}^{\varepsilon}, u_{R}^{\varepsilon}\right)$ with speed $V_{F}$. Once $\operatorname{CD}\left(u_{L}^{\varepsilon}, u_{R}^{\varepsilon}\right)$ reaches $x=\varepsilon$, we apply $\mathrm{RS}_{\mathrm{M}}$ and obtain $\mathrm{NS}\left(u_{L}^{\varepsilon}, \breve{u}_{L}\right)$ and 
Fig. 6 The approximate solution constructed in Example 3


$\mathrm{CD}\left(\check{u}_{L}, \check{u}_{R}\right)$ with speed $V$. Analogously, when $\operatorname{CD}\left(U_{F}, u_{L}^{\varepsilon}\right)$ reaches $x=\varepsilon$, we apply again $\mathrm{RS}_{\mathrm{M}}$ and obtain $\mathrm{NS}\left(U_{F}, \check{U}_{F}\right)$ and $\operatorname{CD}\left(\check{U}_{F}, \check{u}_{L}\right)$ with speed $V$. At last, by letting $\varepsilon \rightarrow 0^{+}$we obtain

$$
\mathrm{RS}_{F}\left[\rho_{L}, \rho_{R}\right](\xi)= \begin{cases}\rho_{L} & \text { if } \xi<\frac{f\left(\rho_{L}\right)-F}{\rho_{L}-R_{F}}, \\ R_{F} & \text { if } \frac{f\left(\rho_{L}\right)-F}{\rho_{L}-R_{F}} \leqslant \xi<0, \\ \check{R}_{F} & \text { if } 0 \leqslant \xi<, \\ \rho_{R} & \text { if } \xi \geqslant V .\end{cases}
$$

Remark 4 Another possible way to deduce $\mathrm{RS}_{F}$ is to consider the microscopic FTL model corresponding to the Cauchy problem for (23) with truncated Riemann datum

$$
\bar{\rho}(x) \doteq \rho_{L} \mathbb{1}_{[-\delta, 0)}(x)+\rho_{R} \mathbb{1}_{[0, \delta]}(x) .
$$

By letting then $n \rightarrow+\infty, \delta \rightarrow+\infty$ and $\varepsilon \rightarrow 0^{+}$we obtain the solution selected by $\mathrm{RS}_{F}$ for Riemann problem (21), (22).

Remark 5 We underline that in [21] it is considered the discontinuous flux (24) with $f_{F}(\rho) \doteq \frac{F}{f_{\max }} f(\rho)$. Even if it differs from our choice, at the limit we obtain the same result. The reason is simply because in any cases we obtain at the limit the Riemann solver $\mathrm{RS}_{\mathrm{M}}$ which maximizes the flow.

Remark 6 Note that a yet different justification of the model of [21] can be given in terms of a velocity constraint; roughly speaking, prescribing limited velocity results in prescribing a specific flux limitation, due to the boundary layer phenomena proper to hyperbolic scalar conservation laws [2, 4, 12, 13, 23]. However this line of justification is beyond the scope of the present paper. 
Acknowledgements The publication has been prepared with the support of the RUDN University Program 5-100.

\section{References}

1. Adimurthi, Mishra, S., Gowda, G.D.V.: Optimal entropy solutions for conservation laws with discontinuous flux-functions. J. Hyperbolic Differ. Equ. 2(4), 783-837 (2005)

2. Amadori, D.: Initial-boundary value problems for nonlinear systems of conservation laws. NoDEA Nonlinear Differ. Equ. Appl. 4(1), 1-42 (1997)

3. Andreianov, B.: The semigroup approach to conservation laws with discontinuous flux. In: Hyperbolic Conservation Laws and Related Analysis with Applications, Springer Proceedings in Mathematics and Statistics, vol. 49, pp. 1-22. Springer, Heidelberg (2014)

4. Andreianov, B.: New approaches to describing admissibility of solutions of scalar conservation laws with discontinuous flux. In: CANUM 2014-42e Congrès National d'Analyse Numérique, ESAIM Proceedings Surveys, vol. 50, pp. 40-65. EDP Sci, Les Ulis (2015)

5. Andreianov, B., Cancès, C.: Vanishing capillarity solutions of Buckley-Leverett equation with gravity in two-rocks' medium. Comput. Geosci. 17(3), 551-572 (2013)

6. Andreianov, B., Cancès, C.: On interface transmission conditions for conservation laws with discontinuous flux of general shape. J. Hyperbolic Differ. Equ. 12(2), 343-384 (2015)

7. Andreianov, B., Donadello, C., Razafison, U., Rosini, M.D.: Qualitative behaviour and numerical approximation of solutions to conservation laws with non-local point constraints on the flux and modeling of crowd dynamics at the bottlenecks. ESAIM Math. Model. Numer. Anal. 50(5), 1269-1287 (2016)

8. Andreianov, B., Donadello, C., Razafison, U., Rosini, M.D.: Analysis and approximation of one-dimensional scalar conservation laws with general point constraints on the flux. J. Math. Pures Appl. 9(116), 309-346 (2018)

9. Andreianov, B., Donadello, C., Rosini, M.D.: Crowd dynamics and conservation laws with nonlocal constraints and capacity drop. Math. Models Methods Appl. Sci. 24(13), 2685-2722 (2014)

10. Andreianov, B., Karlsen, K.H., Risebro, N.H.: A theory of $L^{1}$-dissipative solvers for scalar conservation laws with discontinuous flux. Arch. Ration. Mech. Anal. 201(1), 27-86 (2011)

11. Andreianov, B., Mitrović, D.: Entropy conditions for scalar conservation laws with discontinuous flux revisited. Ann. Inst. H. Poincaré Anal. Non Linéaire 32(6), 1307-1335 (2015)

12. Andreianov, B., Sbihi, K.: Well-posedness of general boundary-value problems for scalar conservation laws. Trans. Am. Math. Soc. 367(6), 3763-3806 (2015)

13. Bardos, C., le Roux, A.Y., Nédélec, J.C.: First order quasilinear equations with boundary conditions. Commun. Partial Differ. Equ. 4(9), 1017-1034 (1979)

14. Benyahia, M., Rosini, M.D.: A macroscopic traffic model with phase transitions and local point constraints on the flow. Netw. Heterog. Media 12(2), 297-317 (2017)

15. Braess, D.: Über ein paradoxon aus der verkehrsplanung. Unternehmensforschung 12(1), 258268 (1968)

16. Bürger, R., Karlsen, K., Risebro, N., Towers, J.: Monotone difference approximations for the simulation of clarifier-thickener units. Comput. Vis. Sci. 6(2), 83-91 (2004)

17. Bürger, R., Karlsen, K.H., Towers, J.D.: An Engquist-Osher-type scheme for conservation laws with discontinuous flux adapted to flux connections. SIAM J. Numer. Anal. 47(3), 1684-1712 (2009)

18. Bürger, R., Karlsen, K.H., Towers, J.D.: On some difference schemes and entropy conditions for a class of multi-species kinematic flow models with discontinuous flux. Netw. Heterog. Media 5(3), 461-485 (2010)

19. Cancès, C.: Asymptotic behavior of two-phase flows in heterogeneous porous media for capillarity depending only on space. I. Convergence to the optimal entropy solution. SIAM J. Math. Anal. 42(2), 946-971 (2010) 
20. Colombo, R.M., Garavello, M.: Phase transition model for traffic at a junction. J. Math. Sci. (N.Y.) 196(1), 30-36 (2014)

21. Colombo, R.M., Goatin, P.: A well posed conservation law with a variable unilateral constraint. J. Differ. Equ. 234(2), 654-675 (2007)

22. Colombo, R.M., Rosini, M.D.: Pedestrian flows and non-classical shocks. Math. Methods Appl. Sci. 28(13), 1553-1567 (2005)

23. Colombo, R.M., Rosini, M.D.: Well posedness of balance laws with boundary. J. Math. Anal. Appl. 311(2), 683-702 (2005)

24. Corli, A., Rosini, M.D.: Coherence and chattering of a one-way valve. ZAMM—J. Appl. Math. Mech./Zeitschrift für Angewandte Mathematik und Mechanik (2019)

25. Dal Santo, E., Donadello, C., Pellegrino, S.F., Rosini, M.D.: Representation of capacity drop at a road merge via point constraints in a first order traffic model. ESAIM Math. Model. Numer. Anal. 53(1), 1-34 (2019)

26. Delle Monache, M.L., Goatin, P., Piccoli, B.: Priority-based Riemann solver for traffic flow on networks. Commun. Math. Sci. 16(1), 185-211 (2018)

27. Di Francesco, M., Fagioli, S., Rosini, M.D.: Deterministic particle approximation of scalar conservation laws. Boll. Unione Mat. Ital. 10(3), 487-501 (2017)

28. Di Francesco, M., Fagioli, S., Rosini, M.D., Russo, G.: Follow-the-leader approximations of macroscopic models for vehicular and pedestrian flows. In: Active Particles. vol. 1. Advances in Theory, Models, and Applications, pp. 333-378. Model. Simul. Sci. Eng. Technol. Birkhäuser/Springer, Cham (2017)

29. Di Francesco, M., Rosini, M.: Rigorous derivation of nonlinear scalar conservation laws from follow-the-leader type models via many particle limit. Arch. Rat. Mech. Anal. 217(3), 831-871 (2015)

30. Di Francesco, M., Fagioli, S., Rosini, M.D.: Many particle approximation of the Aw-RascleZhang second order model for vehicular traffic. Math. Biosci. Eng. 14(1), 127-141 (2017)

31. Di Francesco, M., Fagioli, S., Rosini, M.D., Russo, G.: Deterministic particle approximation of the Hughes model in one space dimension. Kinet. Relat. Models 10(1), 215-237 (2017)

32. Diehl, S.: Continuous sedimentation of multi-component particles. Math. Methods Appl. Sci. 20(15), 1345-1364 (1997)

33. Diehl, S.: A uniqueness condition for nonlinear convection-diffusion equations with discontinuous coefficients. J. Hyperbolic Differ. Equ. 6(1), 127-159 (2009)

34. Garavello, M., Goatin, P.: The Aw-Rascle traffic model with locally constrained flow. J. Math. Anal. Appl. 378(2), 634-648 (2011)

35. Garavello, M., Natalini, R., Piccoli, B., Terracina, A.: Conservation laws with discontinuous flux. Netw. Heterog. Media 2(1), 159-179 (2007)

36. Gimse, T., Risebro, N.H.: Solution of the Cauchy problem for a conservation law with a discontinuous flux function. SIAM J. Math. Anal. 23(3), 635-648 (1992)

37. Hopf, E.: The partial differential equation $u_{t}+u u_{x}=\mu u_{x x}$. Commun. Pure Appl. Math. 3, 201-230 (1950)

38. Kaasschieter, E.F.: Solving the Buckley-Leverett equation with gravity in a heterogeneous porous medium. Comput. Geosci. 3(1), 23-48 (1999)

39. Karlsen, K.H., Risebro, N.H., Towers, J.D.: $L^{1}$ stability for entropy solutions of nonlinear degenerate parabolic convection-diffusion equations with discontinuous coefficients. Skr. K. Nor. Vidensk. Selsk. 3, 1-49 (2003)

40. Kolb, O., Costeseque, G., Goatin, P., Göttlich, S.: Pareto-optimal coupling conditions for the Aw-Rascle-Zhang traffic flow model at junctions. SIAM J. Appl. Math. 78(4), 1981-2002 (2018)

41. Kruzhkov, S.N.: First order quasilinear equations with several independent variables. Mat. Sb. (N.S.) 81 (123), 228-255 (1970)

42. Lax, P.: Shock Waves and Entropy, pp. 603-634 (1971)

43. LeFloch, P.G.: Hyperbolic Systems of Conservation Laws. Lectures in Mathematics ETH Zürich, Birkhäuser Verlag, Basel (2002) 
44. Lighthill, M., Whitham, G.: On kinematic waves. II. A theory of traffic flow on long crowded roads. In: Royal Society of London. Series A, Mathematical and Physical Sciences. vol. 229, pp. 317-345 (1955)

45. Moutari, S., Herty, M., Klein, A., Oeser, M., Steinauer, B., Schleper, V.: Modelling road traffic accidents using macroscopic second-order models of traffic flow. IMA J. Appl. Math. 78(5), $1087-1108$ (2013)

46. Oleĭnik, O.A.: Uniqueness and stability of the generalized solution of the Cauchy problem for a quasi-linear equation. Uspehi Mat. Nauk 14(2(86)), 165-170 (1959)

47. Rayleigh, L.: Aerial plane waves of finite amplitude [Proc. Roy. Soc. London Ser. A 84 (1910), 247-284]. In: Classic papers in shock compression science, pp. 361-404. High-press. Shock Compression Condens. Matter, Springer, New York (1998)

48. Richards, P.I.: Shock waves on the highway. Oper. Res. 4(1), 42-51 (1956)

49. Ross, D.S.: Two new moving boundary problems for scalar conservation laws. Commun. Pure Appl. Math. 41(5), 725-737 (1988)

50. Seguin, N., Vovelle, J.: Analysis and approximation of a scalar conservation law with a flux function with discontinuous coefficients. Math. Models Methods Appl. Sci. 13(2), 221-257 (2003)

51. Shen, W.: Traveling wave profiles for a follow-the-leader model for traffic flow with rough road condition. Netw. Heterog. Media 13(3), 449-478 (2018)

52. Srivastava, A., Geroliminis, N.: Empirical observations of capacity drop in freeway merges with ramp control and integration in a first-order model. Transp. Res. Part C: Emerg. Technol. 30, 161-177 (2013)

53. Towers, J.D.: Convergence of a difference scheme for conservation laws with a discontinuous flux. SIAM J. Numer. Anal. 38(2), 681-698 (2000)

54. Villa, S., Goatin, P., Chalons, C.: Moving bottlenecks for the Aw-Rascle-Zhang traffic flow model. Discrete Contin. Dyn. Syst. Ser. B 22(10), 3921-3952 (2017)

55. Vol'pert, A.I.: Spaces BV and quasilinear equations. Mat. Sb. (N.S.) 73 (115), 255-302 (1967) 